\title{
Metabolic Imaging of Glutamine in Cancer
}

\author{
Lin Zhu ${ }^{1}$, Karl Ploessl², Rong Zhou ${ }^{2}$, David Mankoff ${ }^{2}$, and Hank F. Kung ${ }^{2,3}$ \\ ${ }^{I}$ College of Chemistry 82\#, Beijing Normal University, Beijing, China; ${ }^{2}$ Department of Radiology, University of Pennsylvania School \\ of Medicine, Philadelphia, Pennsylvania; and ${ }^{3}$ Beijing Institute for Brain Disorders, Capital Medical University, Beijing, China
}

\begin{abstract}
Glucose and glutamine are the most abundant nutrients for producing energy and building blocks in normal and tumor cells. Increased glycolysis in tumors, the Warburg Effect, is the basis for ${ }^{18} \mathrm{~F}-\mathrm{FDG}$ PET imaging. Cancer cells can also be genetically reprogrammed to use glutamine. ${ }^{5-11} \mathrm{C}-(2 S)$-glutamine and ${ }^{18} \mathrm{~F}-(2 S, 4 R) 4$-fluoroglutamine may be useful complementary tools to measure changes in tumor metabolism. In glioma patients, the tracer ${ }^{18} \mathrm{~F}-(2 \mathrm{~S}, 4 R) 4$-fluoroglutamine showed tumor-to-background contrast different from that of ${ }^{18} \mathrm{~F}-\mathrm{FDG}$ and differences in uptake in glioma patients with clinical progression of disease versus stable disease (tumor-to-brain ratio $>3.7$ in clinically active glioma tumors, minimal or no specific uptake in clinically stable tumors). These preliminary results suggest that ${ }^{18} \mathrm{~F}-(2 \mathrm{~S}, 4 R) 4$-fluoroglutamine PET may be a new tool for probing in vivo metabolism of glutamine in cancer patients and for guiding glutamine-targeted therapeutics. Further studies of uptake mechanism, and comparison of kinetics for ${ }^{18} \mathrm{~F}-(2 \mathrm{~S}, 4 R) 4$-fluoroglutamine versus the ${ }^{11} \mathrm{C}$-labeled native glutamine, will be important and enlightening.
\end{abstract}

Key Words: glutamine; ${ }^{18} \mathrm{~F}-(2 S, 4 R) 4$-fluoroglutamine; $5-{ }^{11} \mathrm{C}-(2 S)-$ glutamine; cancer metabolism; gliomas; metabolic imaging

J Nucl Med 2017; 58:533-537

DOI: 10.2967/jnumed.116.182345

Cellular metabolism in tumor cells is significantly different from that in normal cells (1). In normal and tumor tissues, glucose is the most common source of nutrient, providing most energy and metabolic substrates for maintaining cell growth. It was $90 \mathrm{y}$ ago that Warburg first reported a dramatic transformation of glucose metabolism and mitochondrial activity occurring in tumor cells. Tumor cells adopt different strategies to survive in a changing microenvironment, forcing the cell to use alternative metabolic processes to support growth and proliferation. Cancer cells modify key metabolic pathways, such as Myc (2), and many other key switching points that control cellular metabolism. Oncogene expression and loss of tumor suppressor gene significantly modify glucose and glutamine metabolism in tumor cells (Fig. 1) $(3,4)$. Epigenetic adaptation in cancer cells plays a critical role in switching different biochemical processes, which lead to increased adenosine triphosphate production through oxidative phosphorylation necessary for tumor cells.

Inhibition of altered metabolic mechanisms is a new strategy for development of cancer therapy $(3,5,6)$. It is now generally accepted that reprogramming of cellular metabolism providing alternative

\footnotetext{
Received Jan. 10, 2017; revision accepted Feb. 17, 2017.

For correspondence or reprints contact: Hank F. Kung, Department of Radiology, University of Pennsylvania School of Medicine, Philadelphia, PA 19104.

E-mail: kunghf@gmail.com

Published online Feb. 23, 2017

COPYRIGHT (C 2017 by the Society of Nuclear Medicine and Molecular Imaging.
}

biochemical machinery to use glutamine is a consequence of oncogenic mutation in promoting tumor growth. Glutamine is the second most abundant nutrient (after glucose) in blood circulation, at a concentration of about 0.5 to $1 \mathrm{mM}$. Recently, there is a renewed interest in investigating cancer metabolism in understanding the mechanisms for tumor proliferation but also as a basis for development of treatments specifically targeting the metabolic pathways sustaining the tumor growth. Such pathways involved in glycolysis or glutaminolysis could be exploited for therapeutic purposes $(1,3)$.

Glutamine is transported across cell membrane by at least 3 different amino acid transporters: sodium-neutral amino acid transporters; alanine, serine, cysteine-preferring transporter 2 (ASCT2, or SLC1A5); and large neutral amino acid transporter 1 (LAT1) (7). These transporters are nonspecific, and they function as transporters for multiple amino acids. The glutamine transporter ASCT2 appeared to be the most prominent and unregulated transporter for glutamine uptake in many cancer cells. Therefore, inhibiting the glutamine uptake by blocking the glutamine transporter ASCT2 is now an active and ongoing area of cancer therapeutic development $(3,8,9)$. After entering into the cell, glutamine is first converted to glutamate by glutaminase (Fig. 1), and subsequently glutamate dehydrogenase removes one more ammonia group from the glutamate to produce $\alpha$-ketoglutarate, through which it enters the tricarboxylic acid cycle for producing metabolic substrates and energy (Fig. 1). Blocking the glutamine transporters or the first metabolic enzyme glutaminase in tumor cells would stop the cancer cells from using glutamine. Inhibitors of glutaminolysis may serve as a therapeutic goal to help starve the cancer to death. The processes may be more selective because of differences in isoforms of glutaminase for tumors versus major metabolic organs such as the liver $(10,11)$.

Currently, PET imaging is an important tool for the diagnosis and monitoring of treatment of cancer. The ${ }^{18} \mathrm{~F}$-FDG PET imaging takes advantage of increased glucose transport and hexokinase II activities of tumor cells-Warburg effect; there is often a significantly increased trapping of ${ }^{18} \mathrm{~F}-\mathrm{FDG}$ in the actively proliferating tumor tissue, which is visualized by PET images. Despite the success of using ${ }^{18}$ F-FDG PET in staging and monitoring tumors in humans, there is a growing realization that some types of active tumors may adapt a different metabolism profile using various metabolic substrates other than glucose.

\section{UPTAKE OF GLUTAMINE IN TUMOR CELL LINES AND TUMOR MODELS}

To develop a glutamine-based PET imaging agent, a convenient procedure to prepare $5-{ }^{11} \mathrm{C}-(2 S)$-glutamine $\left({ }^{11} \mathrm{C}-\mathrm{Gln}\right)$ was reported (Fig. 2A) (12), and this preparation specifically labeled ${ }^{11} \mathrm{C}$ at the $\mathrm{C} 5$ position. Tumor cell uptake studies showed high uptake of ${ }^{11} \mathrm{C}-\mathrm{Gln}$ (reached $17.9 \%$ and $22.5 \%$ per $100 \mu \mathrm{g}$ of protein, respectively, at $60 \mathrm{~min}$ in 9L and SF188 tumor cells). At $30 \mathrm{~min}$ after incubation, 


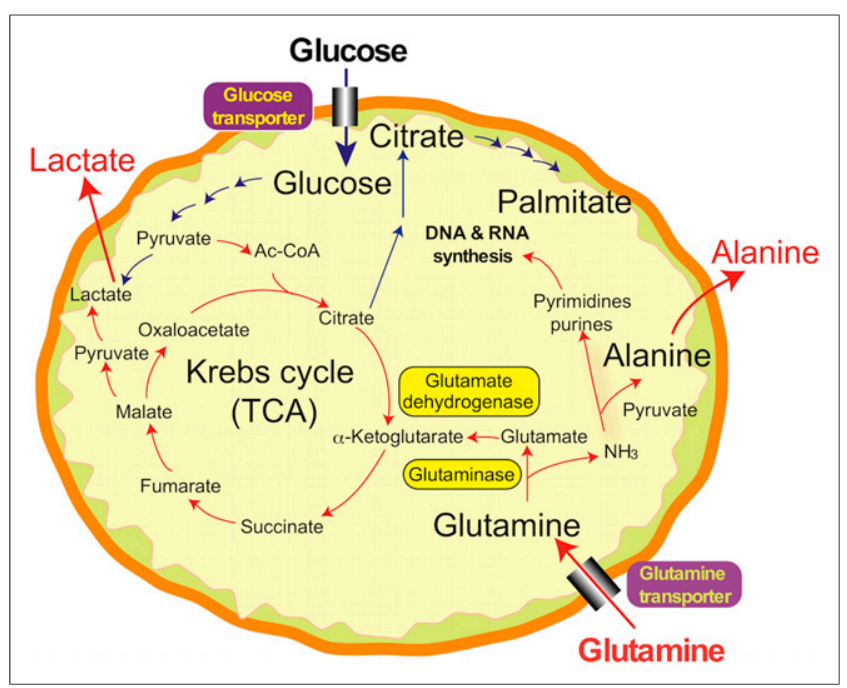

FIGURE 1. Simplified schematic drawing of intracellular metabolism of glucose and glutamine is presented to show possible metabolic changes in tumor cells using glycolysis or glutaminolysis. Tumor cells may use both pathways to generate energy and intermediate metabolites for survival and growth. However, tumor cells may switch energy source to glutamine, and thus enhance their survival and proliferation.

more than $30 \%$ of the activity appeared to be incorporated into cellular protein. The intracellular uptake and metabolism will likely make the quantitative kinetic modeling of ${ }^{11} \mathrm{C}$-Gln in vivo much more complicated, akin to other highly metabolized substrates such as glucose and thymidine (13). Biodistribution in normal mice showed that ${ }^{11} \mathrm{C}-\mathrm{Gln}$ had significant uptake in the pancreas ( $7.37 \%$ injected dose per gram at $15 \mathrm{~min}$ ), most likely because of the exocrine function and high protein turnover within the pancreas. Dynamic small-animal PET studies in rats bearing xenografted 9L tumors and in transgenic mice bearing spontaneous mammary gland tumors showed a prominent tumor uptake and retention. This tracer was favorably taken up in the tumor models. The results suggest that ${ }^{11} \mathrm{C}$-Gln might be useful for probing in vivo tumor metabolism in glutaminolytic tumors (12). Thus far, no human imaging studies of ${ }^{11} \mathrm{C}-\mathrm{G}$ ln have been reported, likely because of the constraint of short physical half-life and complicated radiolabeling and purification steps. However, in the future this agent may still be a useful tool for studying glutamine metabolism of cancer cells and validating the biochemistry of analogs, akin to the use of other ${ }^{11} \mathrm{C}$-labeled substrates to clarify kinetics for commonly used analogs such as thymidine and ${ }^{18} \mathrm{~F}-\mathrm{FDG}(13)$.

Because the half-life of ${ }^{18} \mathrm{~F}(110 \mathrm{~min})$ is 5.5 times longer than that of ${ }^{11} \mathrm{C}$ (20 min), logistically, ${ }^{18} \mathrm{~F}$ tracers are more suited to preparation in off-site cyclotrons and delivery to nuclear medicine clinics via commercial distribution networks currently existing for ${ }^{18}$ F-FDG. A versatile synthetic route to prepare all 4 stereoisomeric ${ }^{19} \mathrm{~F}$ - and ${ }^{18} \mathrm{~F}$-labeled 4-fluoro-glutamines (4-FGln) was developed by exploiting a Passerini 3-component reaction (14). All 4 possible 4-FGln isomers- $(2 S, 4 R),(2 S, 4 S),(2 R, 4 S)$, and $(2 R, 4 R)$ - have been prepared and tested in tumor cell lines. Among them, ${ }^{18} \mathrm{~F}-(2 S, 4 R) 4-$ FGln and ${ }^{18} \mathrm{~F}-(2 S, 4 S) 4-\mathrm{FGln}$ are L-glutamine (natural amino acid) analogs (structures shown in Fig. 2A), whereas $(2 R, 4 S) 4-F G l n$ and $(2 R, 4 R) 4-\mathrm{FGln}$ are $\mathrm{D}$-glutamine (unnatural amino acid) analogs. Only the natural L-glutamine derivatives ${ }^{18} \mathrm{~F}-(2 S, 4 R) 4-\mathrm{FGln}$ and ${ }^{18} \mathrm{~F}-(2 S, 4 S) 4-F G l n$ displayed a significantly higher uptake and retention in tumor cells than ${ }^{18} \mathrm{~F}-\mathrm{FDG}$ and ${ }^{3} \mathrm{H}-(2 S)$-glutamine, suggest- ing that these 2 analogs were preferentially taken up and retained by the tumor cells in vitro under identical condition (Fig. 2B). One isomer, ${ }^{18} \mathrm{~F}-(2 S, 4 R) 4-\mathrm{FGln}$, displayed the most promising properties as a potential diagnostic tracer for PET imaging to assess glutamine transport and distribution in various types of tumors. Additional studies were performed to demonstrate the utility of this tracer. Biodistribution and PET imaging studies showed that ${ }^{18} \mathrm{~F}-(2 S, 4 R)$ 4-FGln localized in tumors with a higher uptake than in surrounding muscle and liver tissues. Significantly, small-animal PET imaging studies of ${ }^{18} \mathrm{~F}-(2 S, 4 R) 4-\mathrm{FGln}$ in 9L tumor xenografts in F344 rats, a well-known and established animal model that represents typical glioblastomas that would be found within a clinical setting, displayed excellent images (Figs. 3 and 4). The imaging studies in this rat tumor model clearly confirmed that the agent is highly selective for tumor. A recent report also suggests that the uptake of ${ }^{18} \mathrm{~F}-(2 S, 4 R) 4-\mathrm{FGln}$, but not ${ }^{18} \mathrm{~F}-\mathrm{FDG}$, correlates with relative ASCT2 levels in xenograft tumors (15). In genetically engineered mice, ${ }^{18} \mathrm{~F}-(2 S, 4 R) 4-F G l n$ accumulation was significantly elevated in lung tumors, relative to normal lung and cardiac tissues (15). In addition, it was reported that cancers can also derive metabolic support from the surrounding stromal cells (16). Enhanced glutamine production in cancer-associated fibroblasts by harnessing carbon and nitrogen to maintain cancer cell growth when glutamine is scarce was observed. Therefore, as a new therapeutic approach it may be possible to cotarget stromal glutamine synthetase and cancer cell glutaminase, which disrupts this metabolic crosstalk and hence induces tumor regression, as shown in an ovarian carcinoma mouse model (16).

It was reported that ${ }^{18} \mathrm{~F}-(2 S, 4 R) 4$-FGln showed a high uptake and retention in tumor tissue, with minimal uptake in the surrounding brain, enabling clear tumor delineation in all glioma animal models

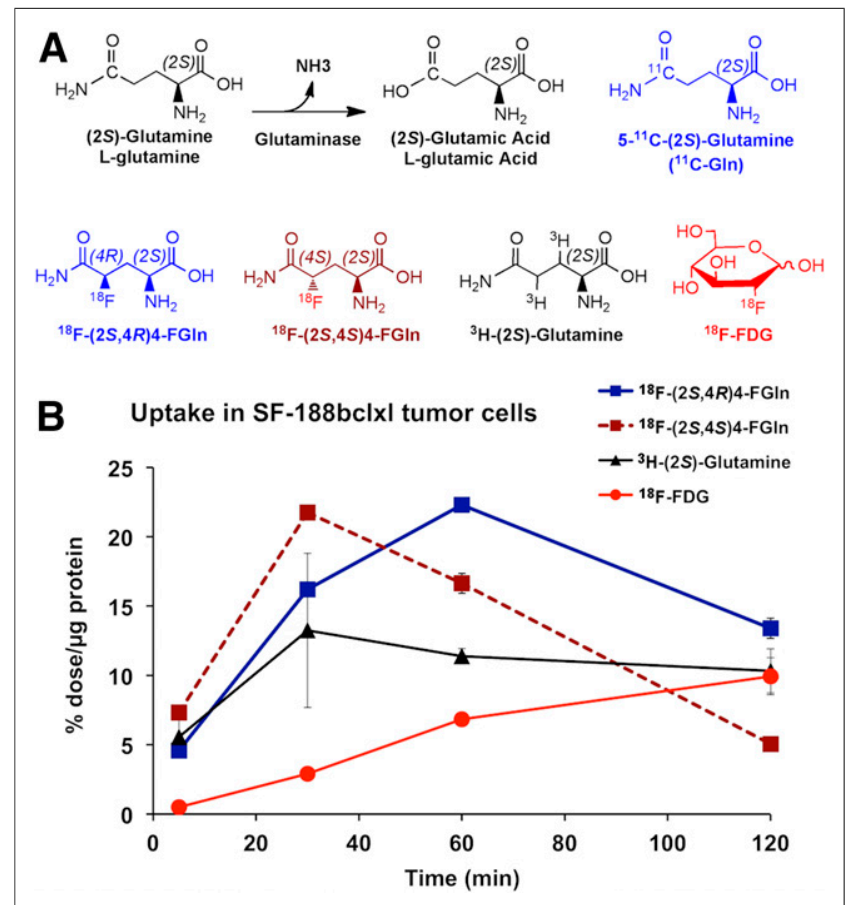

FIGURE 2. (A) Chemical structures of ${ }^{11} \mathrm{C}-\mathrm{Gln},{ }^{18} \mathrm{~F}-(2 \mathrm{~S}, 4 R) 4 \mathrm{~F}-\mathrm{Gln}$, and ${ }^{18} \mathrm{~F}-(2 \mathrm{~S}, 4 \mathrm{~S}) 4 \mathrm{~F}-\mathrm{Gln}$. (Adapted with permission of $(12,14)$.) (B) In vitro uptake studies of ${ }^{18} \mathrm{~F}-(2 S, 4 R) 4-F G l n$ (blue) and ${ }^{18} \mathrm{~F}-(2 S, 4 S) 4-F G l n$ (brown) in SF188bcl-xL cell line. ${ }^{3} \mathrm{H}-\mathrm{L}-$ glutamine (black) and ${ }^{18} \mathrm{~F}-\mathrm{FDG}$ (red) were used as reference ligands. (Modified with permission of (14).) 


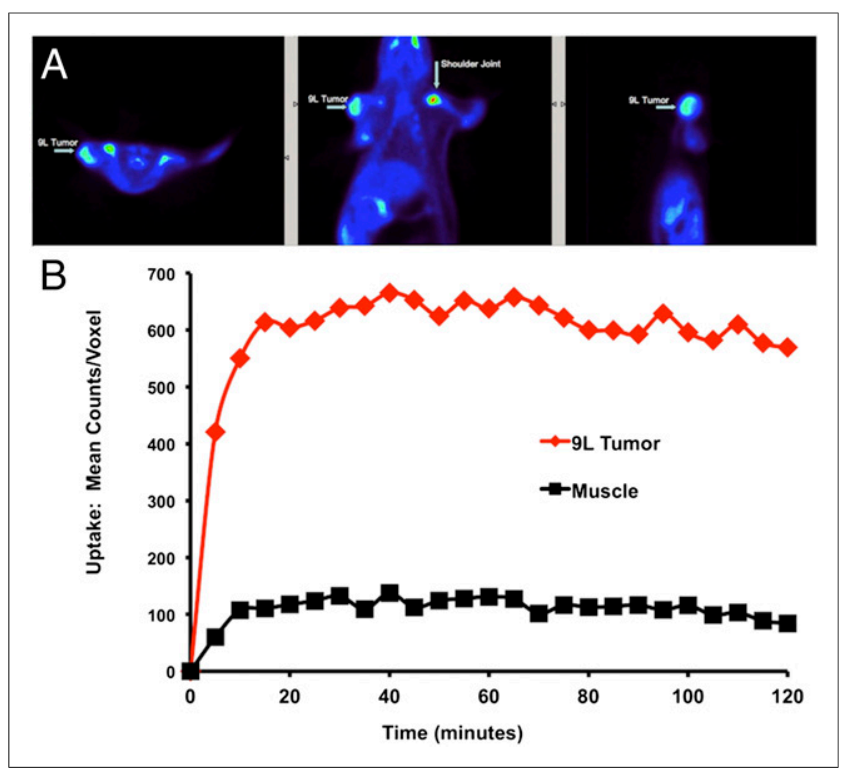

FIGURE 3. (A) Small-animal PET images of ${ }^{18} \mathrm{~F}-(2 S, 4 R) 4$-fluoroglutamine in F344 9L (glioma tumor) rat after intravenous injection. Data represent images from summed 2-h scan. Images are shown in transverse, coronal, and sagittal views. Arrows represent location of tumors. (B) Small-animal PET time-activity curve for ${ }^{18} \mathrm{~F}$ - $(2 \mathrm{~S}, 4 R) 4$-fluoroglutamine after intravenous injection into F344 rat bearing xenografted $9 \mathrm{~L}$ tumor on left shoulder. (Modified from reference (31).)

tested (17). No ${ }^{18} \mathrm{~F}-(2 S, 4 R) 4-F G l n$ uptake was detected in a model of impaired blood-brain barrier or multiple animal models of neuroinflammation. The results suggest that ${ }^{18} \mathrm{~F}-(2 S, 4 R) 4-\mathrm{FGln}$ uptake is mainly mediated by the amino acid transporter ASCT2, which was minimally expressed in the normal brain but markedly increased in gliomas (18).

There is a vast difference in biologic behavior between glutamine and glutamic acid analogs $\left({ }^{18} \mathrm{~F}-(2 S, 4 R) 4-\mathrm{FGln}\right.$ vs. ${ }^{18} \mathrm{~F}-(2 S, 4 R) 4-$ FGlu) (19). Imaging studies showed that both tracers had fast accumulation in 9L tumors. ${ }^{18} \mathrm{~F}-(2 S, 4 R) 4-F G l n$ displayed a prolonged retention in the tumor model, whereas the glutamic acid derivative ${ }^{18} \mathrm{~F}-(2 S, 4 R) 4-F G l u$ exhibited fast efflux from the tumor tissue. It is reasonable to conclude that ${ }^{18} \mathrm{~F}-(2 S, 4 R) 4-\mathrm{FGlu}$ enters tumor cells with high efficiency with a low level of metabolism inside the cells. Converging evidence suggests that $(2 S, 4 R) 4$-FGln is not a good substrate for different forms of glutaminase, further supporting the inertness of this fluorinated glutamine as substrate for further metabolism (20) and as a probe for glutamine transport and intracellular pool size.

Because glutaminolysis is an alternative metabolic pathway, many aggressive cancers under stressful microenvironments may adopt it to overcome limitations on nutrients and oxidative potential-limiting tumor growth. One such example is the triple-negative breast cancer (TNBC; refers to any breast cancer that does not express the genes for estrogen receptor, progesterone receptor, and human epidermal growth factor receptor 2), which used glutamine via reprogramming gene expression for survival and growth. Xenografts of human TNBC cells and estrogen receptor-positive breast cancer (MCF7 tumor cells) in mice exhibited either high or low glutaminase activity, respectively. It was demonstrated that the enzyme activities in these tumor models can be monitored using ${ }^{18} \mathrm{~F}-(2 S, 4 R) 4$ FGln PET imaging before and after treatment with 2 glutaminase inhibitors: BPTES (bis-2-(5-phenylacetamido-1,2,4-thiadiazol-2- yl)ethyl sulfide) and CB-839 ( $N$-[5-[4-[6-[[2-[3-(trifluoromethoxy) phenyl] acetyl] amino]-3-pyridazinyl]butyl]-1,3,4-thiadiazol2-yl]-2-pyridineacetamide, 2-(pyridin-2-yl)- $N$-(5-(4-(6-(2-(3(trifluoromethoxy) phenyl)acetamido)pyridazin-3-yl)butyl)1,3,4-thiadiazol-2-yl)acetamide), or vehicle solution. The tumor-to-blood activity ratios (T/B) were obtained from PET images and compared with tumor glutamine concentrations $(\mu \mathrm{mol} / \mathrm{g})$ assayed by highresolution ${ }^{1} \mathrm{H}$ MR spectroscopy of tumor homogenates. Results from this study suggested an interesting interplay between intracellular glutamine concentration and glutaminase enzyme inhibition. It was apparent that because of differential glutaminase activities, TNBC tumors that actively catabolize glutamine exhibited a distinctly lower glutamine concentration compared with MCF7 (a cell type with minimal glutaminolysis) at baseline. On glutaminase inhibition, glutamine concentration increased only in TNBC: The T/B values increased significantly compared with baseline, and the percentage change of $\mathrm{T} / \mathrm{B}$ in the glutaminase inhibitor-treated group differed significantly compared with the vehicle-treated group. In contrast, no significant change in T/B was detected in MCF7 tumors after glutaminase inhibitor treatment compared either with baseline or with vehicle treatment. Across both tumor types, there was a positive correlation between T/B values (noninvasively by PET) versus tumor glutamine concentrations estimated by MR spectroscopy. Within the time frame of PET imaging $(1 \mathrm{~h}),{ }^{18} \mathrm{~F}-(2 S, 4 R) 4-\mathrm{FGln}$ was minimally metabolized and remained primarily as the parent ligand in the tumor and blood. This example demonstrates that in breast cancers with high glutaminase activity, an increase of cellular glutamine pool size induced by glutaminase inhibitors can be

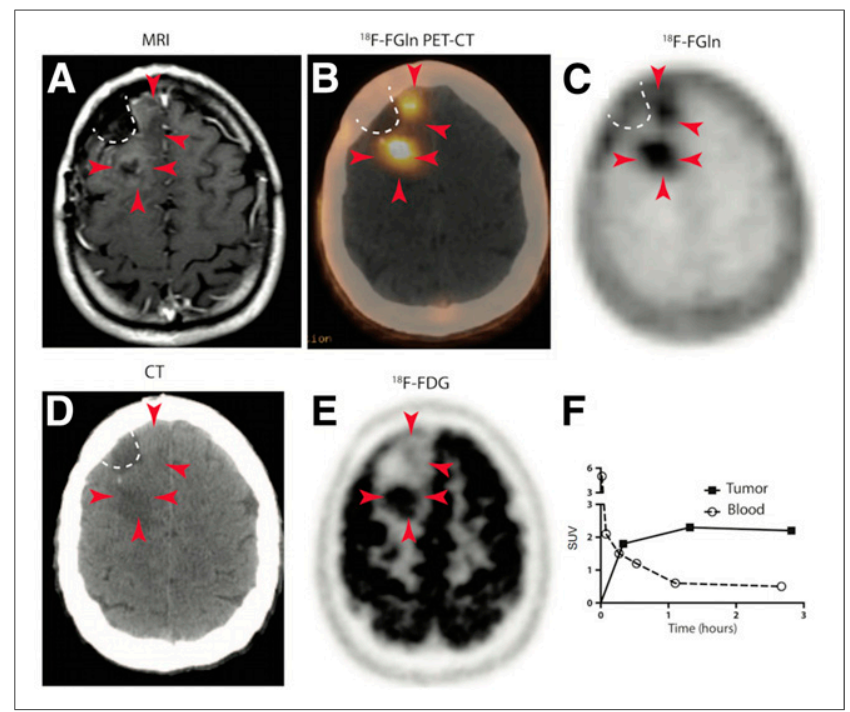

FIGURE 4. ${ }^{18} \mathrm{~F}-4-\mathrm{FG}$ ln shows uptake in human gliomas undergoing progression. (A-F) Images from glioma patient. (A) T1-weighted MR image with contrast enhancement from a 42-y-old IDH1m (isocitrate dehydrogenase 1 mutation) oligodendroglioma patient showing tumor with minimal gadolinium enhancement (red arrows) along surgical cavity (indicated by white dotted line). (B) Fusion ${ }^{18} \mathrm{~F}-4-\mathrm{FGIn} \mathrm{PET} / \mathrm{CT}$ showing ${ }^{18} \mathrm{~F}-4-\mathrm{FG}$ in uptake in areas corresponding to tumor (red arrows). (C) ${ }^{18} \mathrm{~F}-4-\mathrm{FG}$ In PET showing high uptake in tumor with minimal uptake in surrounding brain. (D) CT scan used to generate PET/CT fusion image in B. (E) ${ }^{18} \mathrm{~F}-\mathrm{FDG}$ PET image from same patient showing high background brain avidity and tumor uptake in posterior part of tumor (3 red arrows), but not in anterior portion (2 red arrows). (F) Time-activity curve of ${ }^{18} \mathrm{~F}-4-\mathrm{FGIn}$ : SUVs corresponding to tumor (black squares) and blood (clear circles). (Reprinted with permission of (17).) 
sensitively detected by PET imaging with ${ }^{18} \mathrm{~F}-(2 S, 4 R) 4-\mathrm{FGln}$. It is also suggested that ${ }^{18} \mathrm{~F}-(2 S, 4 R) 4-F G l n$ may be useful for assessing the pharmacodynamics of drugs targeting this cancer-specific glutamine metabolism (18). The increased glutaminase level in these cells is by no means an accident; the TNBC cells may have undergone epigenetic reprogramming in response to changes in the microenvironment. Inhibition of this enzyme in this type of tumor growth may be monitored by PET imaging with ${ }^{18} \mathrm{~F}-(2 S, 4 R) 4-$ FGln. Additional studies using $5-{ }^{11} \mathrm{C}-(2 S)$-glutamine, suitable for intracellular metabolism, may likely lead to avid contrast for glutamine use in these tumor cells. However, caution should be taken that the apparent differences between gliomas and breast cancers may, in part, be due to the impact of both specific and nonspecific transport across the blood-brain barrier in gliomas. Other metabolic cancers also show abnormal glutamine metabolism, including several Krebs cycle-related endocrine tumors (pheochromocytoma and renal cell carcinoma) $(21,22)$. These cancers are related to SDHB and FH mutations that result in abnormal glycolysis and presumably glutaminolysis working in symbiosis in these cancers.

\section{IMAGING STUDIES IN HUMANS}

In the past few years, efforts in investigation of ${ }^{18} \mathrm{~F}-(2 S, 4 R) 4-$ FGln in cancer patients have led to an improved understanding of glutamine function in humans and its potential application in cancer patients $(17,23)$, especially in brain tumor patients in whom high ${ }^{18} \mathrm{~F}$-FDG uptake in normal brain tissue interferes with the detection of specific tumor uptake. Recently, Venneti et al. reported that in glioma patients the new tracer appears to show unique features different from those of ${ }^{18} \mathrm{~F}-\mathrm{FDG}$. Comparison of ${ }^{18} \mathrm{~F}-(2 S, 4 R) 4-$ FGln uptake in glioma patients with clinical progression of disease and patients with stable disease showed minimal ${ }^{18} \mathrm{~F}-(2 S, 4 R) 4-$ FGln uptake in normal brain parenchyma, and the ${ }^{18} \mathrm{~F}-(2 S, 4 R) 4-$ FGln retention in all tumors showed T/Bs between 3.7 and 4.8. In contrast, clinically stable tumors showed minimal or no ${ }^{18} \mathrm{~F}-(2 S, 4 R) 4-\mathrm{FGln}$ activity on PET. Normal brain tissues in these same patients demonstrated high ${ }^{18}$ F-FDG activity, with normal ${ }^{18} \mathrm{~F}-\mathrm{FDG}$ brain concentrations (SUV) equivalent to or greater than tumor SUVs (T/B range, 0.9-1.0). Images of ${ }^{18} \mathrm{~F}-\mathrm{FDG}$ could distinguish the posterior portion of the tumor (Fig. 4E, 3 red arrows) from the surrounding brain, but not the anterior part ( 2 red arrows, Fig. 4E). In contrast, both regions of the tumor showed high uptake with ${ }^{18} \mathrm{~F}-(2 S, 4 R) 4-F G l n$ (Fig. 4C). The infiltrative nature of gliomas may be the cause of this observation. Further, this patient's tumor demonstrated mild contrast enhancement on gadoliniumenhanced MRI (Fig. 4A) but high ${ }^{18} \mathrm{~F}-(2 S, 4 R) 4-F G l n$ avidity (Fig. 4C) and retention of ${ }^{18} \mathrm{~F}-(2 S, 4 R) 4-F G l n$ compared with its rapid clearance in the blood (Fig. $4 \mathrm{~F}$ ). These findings in human subjects demonstrate that clinical ${ }^{18} \mathrm{~F}-(2 S, 4 R) 4$-FGln PET can evaluate high-grade glioma in vivo and may be potentially useful in identifying tumors undergoing transformation. It addition, it may also provide a unique tool for studying the progression, monitoring tumors after radiation and chemotherapy in glioma patients. Further studies will be needed to elucidate the kinetics of ${ }^{18} \mathrm{~F}-(2 S, 4 R) 4-\mathrm{FGln}$ in brain tumors and to understand the relative importance of transport and cellular pool size in determining uptake seen on PET.

\section{OTHER AMINO ACID DERIVATIVES FOR TUMOR IMAGING}

Several ${ }^{11} \mathrm{C}$ - and ${ }^{18} \mathrm{~F}$-labeled amino acids have been used as PET tumor imaging agents in humans (24). These include L- ${ }^{11} \mathrm{C}-m e t h i o-$ nine, ${ }^{\mathrm{L}-18}{ }^{18} \mathrm{~F}$-fluoro- $\alpha$-methyl-tyrosine, $O-\left(2-{ }^{18} \mathrm{~F}\right.$-fluoroethyl $)$-tyrosine $\left({ }^{18} \mathrm{~F}-\mathrm{FET}\right)$, and anti-1-amino-3- ${ }^{18} \mathrm{~F}$-fluorocyclobutylcarboxylic acid (fluciclovine, axumin) (25). Uptake of these tracers permits imaging of primary and metastatic prostate cancer and is likely related to the increased expression of amino acid transporters in tumors. Compared with ${ }^{18} \mathrm{~F}$-FDG, ${ }^{18} \mathrm{~F}$-fluciclovine shows a low renal excretion. Apparently, once it is transported across the membrane, no further metabolism occurs. Another labeled amino acid derivative, ${ }^{18} \mathrm{~F}-\mathrm{FET}$, is a tyrosine analog that is transported across the cell membrane via LAT and is not incorporated into cellular proteins. In contrast to ${ }^{18} \mathrm{~F}-\mathrm{FDG}$ and methionine, it is not taken up in inflammatory cells. As such, it is often used for imaging brain tumors such as gliomas. The mechanism of uptake of ${ }^{18}$ F-FET is not related to glutamine use but rather to the upregulation of amino acid transporter, LAT, at the tumor cell membrane. A recent report showed that 3-fluoropropyl analogs of glutamine, namely ${ }^{18} \mathrm{~F}-(2 S, 4 R)$ - and ${ }^{18} \mathrm{~F}-(2 S, 4 S)$-4-(3-fluoropropyl) glutamine, have in vitro and in vivo tumor-specific uptake: they displayed cell uptake efficiently in 9L tumor cells, with a steady increase over a time frame of $120 \mathrm{~min}$. The in vitro cell uptake studies also suggested that ${ }^{18} \mathrm{~F}-(2 S, 4 S)$-4-(3-fluoropropyl)glutamine is most sensitive to the LAT. In vivo PET imaging studies demonstrated tumorspecific uptake in rats bearing $9 \mathrm{~L}$ xenographs. However, the tumor uptake and retention mechanisms may be significantly different from other glutamine probes, such as ${ }^{11} \mathrm{C}-\mathrm{Gln}$ and ${ }^{18} \mathrm{~F}-(2 S, 4 R)-4-\mathrm{FGln}$ (26). Amino acid Xc transporter system is an active transporter for negatively charged amino acids, such as glutamic acid. This transporter, Xc system, differs from glutamine transporters because glutamine is a neutral amino acid and transported across cell membrane via 3 different neutral amino acid transporters (27). (4S)-4-(3- ${ }^{18}$ F-fluoropropyl)-L-glutamate (BAY94-9392) is an L-glutamate derivative that is specifically taken up by system Xc in tumor models and cancer patients (27-29). A similar tracer targeting the Xc transporter system, 5- ${ }^{18}$ F-fluoro-aminosuberic acid, also demonstrated potential usefulness as a tracer for monitoring of upregulation of system Xc transporter and oxidative stress (30). Currently reported ${ }^{18} \mathrm{~F}$-labeled amino acid-based PET imaging agents are designed to take advantage of the increase of amino acid transporters on the membrane of tumor tissue. However, they are unlikely to be specific for detecting changes in glutamine metabolism in tumor cells.

\section{PERSPECTIVES}

In summary, the glutamine tracers $5-{ }^{11} \mathrm{C}-(2 S)$-glutamine $\left({ }^{11} \mathrm{C}-\mathrm{Gln}\right)$ and ${ }^{18} \mathrm{~F}-(2 S, 4 R) 4$-fluoroglutamine $\left({ }^{18} \mathrm{~F}-(2 S, 4 R) 4-\mathrm{FGln}\right)$ are useful for probing in vivo metabolism of glutamine in cancer cells. It is likely that ${ }^{11} \mathrm{C}$-Gln enters the tumor cells and converts to glutamic acid by glutaminase and subsequently burns up as fuel in the tricarboxylic acid cycle. On the other hand, ${ }^{18} \mathrm{~F}-(2 S, 4 R) 4-\mathrm{FGln}$ may enter tumor cells efficiently and is trapped in cytosol with minimal in vivo metabolism. Both tracers may be useful for probing the epigenetic changes in various tumors and monitoring effects of radiation and chemotherapy in patients. Apparent differences between glioma and somatic tumor models (breast cancer xenografts) suggest that the kinetics and biologic determinants of glutamine probe uptake need further studies, and a comparison of kinetics of the true substrate $\left({ }^{11} \mathrm{C}-\mathrm{Gln}\right)$ to the nonmetabolized analog $\left({ }^{18} \mathrm{~F}-(2 S, 4 R) 4-\mathrm{FGln}\right)$ is likely to be important in understanding in vivo biochemistry, as was the case for glucose probes. Many different pathways linked to changes of tumor metabolism are being explored as targets with the goal of starving the tumor cells to death. Molecular imaging based on glutamine metabolism may provide useful tools for further 
understanding tumor metabolism as well as development of novel therapies to reverse tumor growth.

\section{DISCLOSURE}

This work was supported in part by grants from Stand-Up 2 Cancer (SU2C), PA Health Department, and National Institutes of Health (RO1-CA-164490 and RO1-CA-211337). No other potential conflict of interest relevant to this article was reported.

\section{ACKNOWLEDGMENT}

We thank Dr. Seok Rye Choi for editorial assistance.

\section{REFERENCES}

1. Pavlova NN, Thompson CB. The emerging hallmarks of cancer metabolism. Cell Metab. 2016;23:27-47.

2. Hsieh AL, Dang CV. MYC, metabolic synthetic lethality, and cancer. Recent Results Cancer Res. 2016;207:73-91.

3. Altman BJ, Stine ZE, Dang CV. From Krebs to clinic: glutamine metabolism to cancer therapy. Nat Rev Cancer. 2016;16:619-634.

4. Hensley CT, Wasti AT, DeBerardinis RJ. Glutamine and cancer: cell biology, physiology, and clinical opportunities. J Clin Invest. 2013;123:3678-3684.

5. Kishton RJ, Rathmell JC. Novel therapeutic targets of tumor metabolism. Cancer J. 2015;21:62-69.

6. Martinez-Outschoorn UE, Peiris-Pages M, Pestell RG, Sotgia F, Lisanti MP. Cancer metabolism: a therapeutic perspective. Nat Rev Clin Oncol. 2017;14: 11-31.

7. Bröer A, Rahimi F, Broer S. Deletion of amino acid transporter ASCT2 (SLC1A5) reveals an essential role for transporters SNAT1 (SLC38A1) and SNAT2 (SLC38A2) to sustain glutaminolysis in cancer cells. J Biol Chem. 2016;291:13194-13205.

8. Wang Q, Hardie RA, Hoy AJ, et al. Targeting ASCT2-mediated glutamine uptake blocks prostate cancer growth and tumour development. J Pathol. 2015;236: 278-289.

9. Schulte ML, Khodadadi AB, Cuthbertson ML, Smith JA, Manning HC. 2-amino-4bis(aryloxybenzyl)aminobutanoic acids: a novel scaffold for inhibition of ASCT2mediated glutamine transport. Bioorg Med Chem Lett. 2016;26:1044-1047.

10. Li Y, Erickson JW, Stalnecker CA, et al. Mechanistic basis of glutaminase activation: a key enzyme that promotes glutamine metabolism in cancer cells. J Biol Chem. 2016;291:20900-20910.

11. Stine ZE, Dang CV. Q-ing tumor glutaminase for therapy. Oncotarget. 2015;6: 38440-38441.

12. Qu W, Oya S, Lieberman BP, et al. Preparation and characterization of L-[5- $\left.{ }^{11} \mathrm{C}\right]$-glutamine for metabolic imaging of tumors. J Nucl Med. 2012;53: 98-105.
13. Krohn KA, Mankoff DA, Muzi M, Link JM, Spence AM. True tracers: comparing FDG with glucose and FLT with thymidine. Nucl Med Biol. 2005;32:663-671.

14. Qu W, Zha Z, Ploessl K, et al. Synthesis of optically pure 4-fluoro-glutamines as potential metabolic imaging agents for tumors. J Am Chem Soc. 2011;133:1122-1133.

15. Hassanein M, Hight MR, Buck JR, et al. Preclinical evaluation of $4-{ }^{18} \mathrm{~F}$-fluoroglutamine PET to Assess ASCT2 expression in lung cancer. Mol Imaging Biol. 2016;18:18-23.

16. Yang L, Achreja A, Yeung TL, et al. Targeting stromal glutamine synthetase in tumors disrupts tumor microenvironment-regulated cancer cell growth. Cell Metab. 2016;24:685-700.

17. Venneti S, Dunphy MP, Zhang H, et al. Glutamine-based PET imaging facilitates enhanced metabolic evaluation of gliomas in vivo. Sci Transl Med. 2015;7: 274 ra17.

18. Zhou R, Pantel AR, Li S, et al. ${ }^{18} \mathrm{~F}(2 \mathrm{~S}, 4 \mathrm{R}) 4$-fluoroglutamine PET detects glutamine pool size changes in triple-negative breast cancer in response to glutaminase inhibition. Cancer Res. 2017;77:1476-1484.

19. Ploessl K, Wang L, Lieberman BP, Qu W, Kung HF. Comparative evaluation of ${ }^{18} \mathrm{~F}$-labeled glutamic acid and glutamine as tumor metabolic imaging agents. $J$ Nucl Med. 2012;53:1616-1624.

20. Cooper AJ, Krasnikov BF, Pinto JT, Kung HF, Li J, Ploessl K. Comparative enzymology of (2S,4R)4-fluoroglutamine and (2S,4R)4-fluoroglutamate. Comp Biochem Physiol B Biochem Mol Biol. 2012;163:108-120.

21. Linehan WM, Ricketts CJ. The metabolic basis of kidney cancer. Semin Cancer Biol. 2013;23:46-55.

22. Jochmanova I, Pacak K. Pheochromocytoma: the first metabolic endocrine cancer. Clin Cancer Res. 2016;22:5001-5011.

23. Kim MM, Parolia A, Dunphy MP, Venneti S. Non-invasive metabolic imaging of brain tumours in the era of precision medicine. Nat Rev Clin Oncol. 2016;13:725-739.

24. Huang C, McConathy J. Radiolabeled amino acids for oncologic imaging. J Nucl Med. 2013;54:1007-1010.

25. Schuster DM, Nanni C, Fanti S. Evaluation of prostate cancer with radiolabeled amino acid analogs. $J$ Nucl Med. 2016;57:61S-66S.

26. Wu Z, Zha Z, Li G, et al. ${ }^{18} \mathrm{~F}-(2 \mathrm{~S}, 4 \mathrm{~S})-4-(3$-fluoropropyl)glutamine as a tumor imaging agent. Mol Pharm. 2014;11:3852-3866.

27. Mosci C, Kumar M, Smolarz K, et al. Characterization of physiologic ${ }^{18}$ F-FSPG uptake in healthy volunteers. Radiology. 2016;279:898-905.

28. Kavanaugh G, Williams J, Morris AS, et al. Utility of $\left[{ }^{18} \mathrm{~F}\right] \mathrm{FSPG}$ PET to image hepatocellular carcinoma: first clinical evaluation in a US population. Mol Imaging Biol. 2016;18:924-934.

29. Mittra ES, Koglin N, Mosci C, et al. Pilot preclinical and clinical evaluation of (4S)-4-(3-( ${ }^{18} \mathrm{~F}$-fluoropropyl)-L-glutamate $\left({ }^{18} \mathrm{~F}\right.$-FSPG) for PET/CT imaging of intracranial malignancies. PLoS One. 2016;11:e0148628.

30. Yang H, Jenni S, Colovic M, et al. ${ }^{18} \mathrm{~F}-5$-fluoro-aminosuberic acid (FASu) as a potential tracer to gauge oxidative stress in breast cancer models. J Nucl Med. October 27, 2016 [Epub ahead of print].

31. Lieberman BP, Ploessl K, Wang L, et al. PET imaging of glutaminolysis in tumors by ${ }^{18} \mathrm{~F}-(2 \mathrm{~S}, 4 \mathrm{R}) 4-$ fluoroglutamine. J Nucl Med. 2011;52:1947-1955. 